\title{
Calcul des indices d'humidité des sols dans le bassin versant du Gardon d'Anduze à partir des données météorologiques
}

\author{
Calculation of soil moisture indexes \\ in the Gardon d'Anduze \\ catchment area from meteorological data
}

\author{
Pierre Rascle
}

Ingénieur en Chef des Ponts-et-Chaussées

Ex-Directeur Adjoint de l'Agence de Bassin

Rhône Méditerranée Corse

La présente étude a pour objet de chercher à établir par le calcul les indices d'humidité d'un sol à partir de la pluie et de l'évaporation observées.

Les données utilisées font partie d'un ensemble de données recueillies par l'Agence de Bassin Rhône Méditerranée - Corse à l'occasion de l'élaboration d'un modèle de prévision des crues dans le bassin versant du Gardon d'Anduze. Ce modèle a fait l'objet d'une thèse publiée en 1974 par M. Hofer.

Parmi les données recueillies dans cette haute vallée des Cévennes, intéressant un bassin versant de $545 \mathrm{~km}^{2}$, nous disposions :

- d'observations journalières de pluie et d'évaporation,

- de mesures hebdomadaires d'indices d'humidité, mesures effectuées à l'aide d'un carrotage dans les $20 \mathrm{~cm}$ supcrficiels du sol,

et il a semblé intéressant d'examiner s'il existait une corrélation entre ces deux séries d'observations.

Il faut donc signaler dès à présent que ces calculs d'indice d'humidité conduisent seulement à la connaissance de l'humidité superficielle du sol.

Les recherches ont porté sur 3 parcelles dénommées :

- Le POMPIDOU (terrain schisteux)

- PALLIERES (terrain calcaire)

- CADERLE (terrain granitique)

Les paramètres pluie et évaporation pris en compte sont les moyennes arithmétiques des valeurs observées sur les stations voisines (trois ou quatre en général). Lorsque les mesures d'évaporation faisaient défaut, celle-ci a été déterminée par la méthode Thornthwaite.

\section{Description de la méthode utilisée}

Nous avons comparé sur des périodes de l'ordre de 4 ans : 1970-1974 (correspondant à la durée des observations), les indices mesurés à des indices calculés. Le calcul a été effectué en prenant comme base de départ le premier indice mesuré et en effectuant ensuite des corrections journalières d'indice tenant compte des quantités de pluie et d'évaporation observées, chaque indice journalier calculé étant pris comme base pour la détermination de l'indice journalier suivant.

Le calcul de la correction journalière d'indice consiste à prendre en compte chaque jour :

- une pluie "efficace" (conduisant à une augmentation d'humidité) par application à la quantité de pluie d'un coefficient fonction de l'indice d'humidité au moment considéré,

-- une évaporation "efficace" (conduisant à une diminution d'humidité) par application à l'évaporation observée d'un autre coefficient fonction aussi de l'indice d'humidité.

Les indices d'humidité peuvent varier pour les parcelles considérées entre les limites indiquées ci-après:

\begin{tabular}{|l|c|c|}
\hline PARCELLE & $\begin{array}{c}\text { Indice de } \\
\text { flétrissement } I_{f}\end{array}$ & $\begin{array}{c}\text { Indice de } \\
\text { saturation } /_{s}\end{array}$ \\
\hline LE POMPIDOU & 8,5 & 39 \\
PALLIERES & 15 & 40 \\
CADERLE & 6 & 20 \\
\hline
\end{tabular}

Il est évident que pour un sol à l'indice de saturation, une pluie nouvelle n'apportera pas d'augmentation d'humidité, alors que l'évaporation sera efficace et que le phénomène contraire sera observé dans le cas d'un sol à l'indice de flétrissement.

Pour les 3 parcelles considérées, les coefficients à appliquer à la pluie et à l'évaporation en fonction de l'indice d'humidité sont donnés par des courbes ayant les allures générales indiquées sur la figure 1 . 


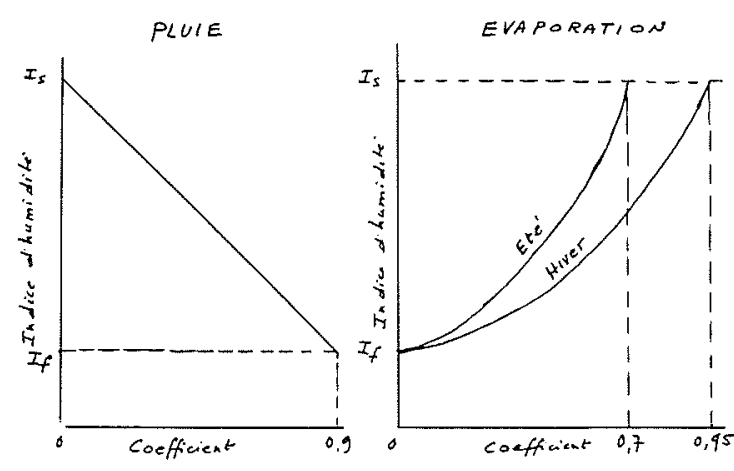

Figure 1

Ces courbes ont été déterminées par tatonnements et ont été mises en équation avec un certain nombre de paramètres qui permettent d'en modifier l'allure dans une recherche concernant un sol déterminé.

Les données fixes sont les deux indices:

\section{saturation}

flétrissement

Les paramètres variables sont :

- les coefficients limites pour la pluie : 0,9 pour l'évaporation : 0,7 en été 0,95 en hiver

- un coefficient qui peut donner à la courbe une forme plus ou moins concave

- le coefficient qui transforme les épaisseurs de pluie et d'évaporation efficaces en points d'indice d'humi- dité. Ce coefficient est à déterminer par tatonnements. Pour les 3 parcelles en cause, il a été pris égal à $1 / 200$ de l'indice de saturation. C'est une première méthode d'approche. Une seconde méthode consiste à considérer que la pluie et l'évaporation efficaces intéressent une tranche de sol de l'ordre de 50 à $60 \mathrm{~cm}$ d'épaisseur et à déterminer le coefficient de transformation, en prenant en compte le poids du sol sec sur cette épaisseur.

Il faut signaler enfin que, dans les calculs, les pluies ont été écrétées à $30 \mathrm{~mm} / \mathrm{jour}$.

Les périodes appelées Eté et Hiver sont limitées par les dates du 15 avril et du 15 septembre.

\section{Résultats obtenus}

Nous donnons à titre d'exemple, figures 2 et 3 , les graphiques obtenus pour l'année 1972 sur les parcelles de PALLIERES et de CADERLE. Sur ces graphiques, nous n'avons pas, par simplification, fait figurer tous les indices calculés, mais seulement ceux correspondant aux jours où des mesures d'indice étaient effectuées.

Au terme des 4 ans, il a été constaté que les écarts sont de 0,3 point d'indice pour la parcelle Le POMPIDOU, 7 points pour PALLIERES et 2,1 points pour CADERLE. Ils atteignent des valeurs plus importantes à certaines périodes, mais ces écarts se corrigent dans le temps et les écarts moyens sont relativement faibles.

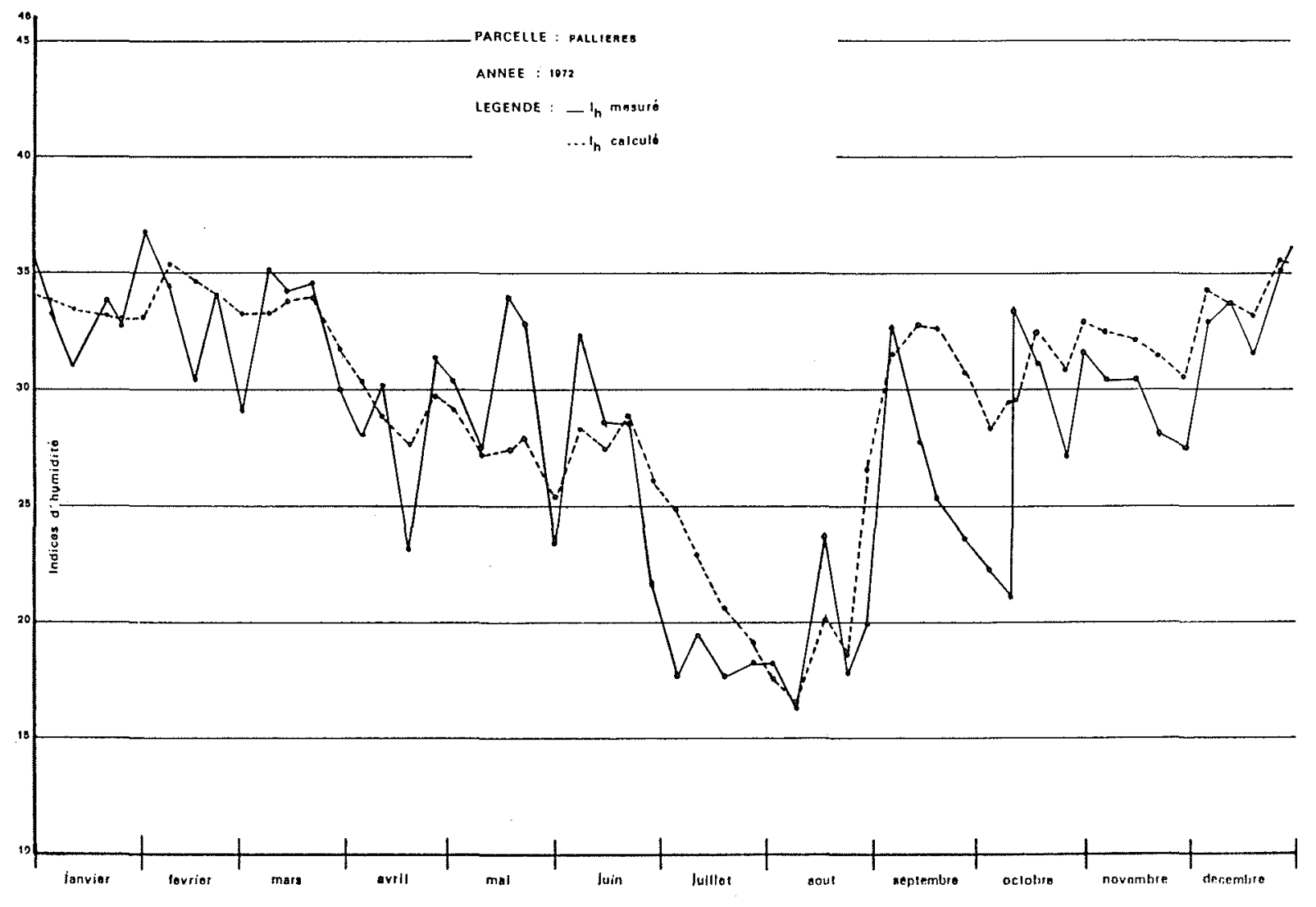

Figure 2 


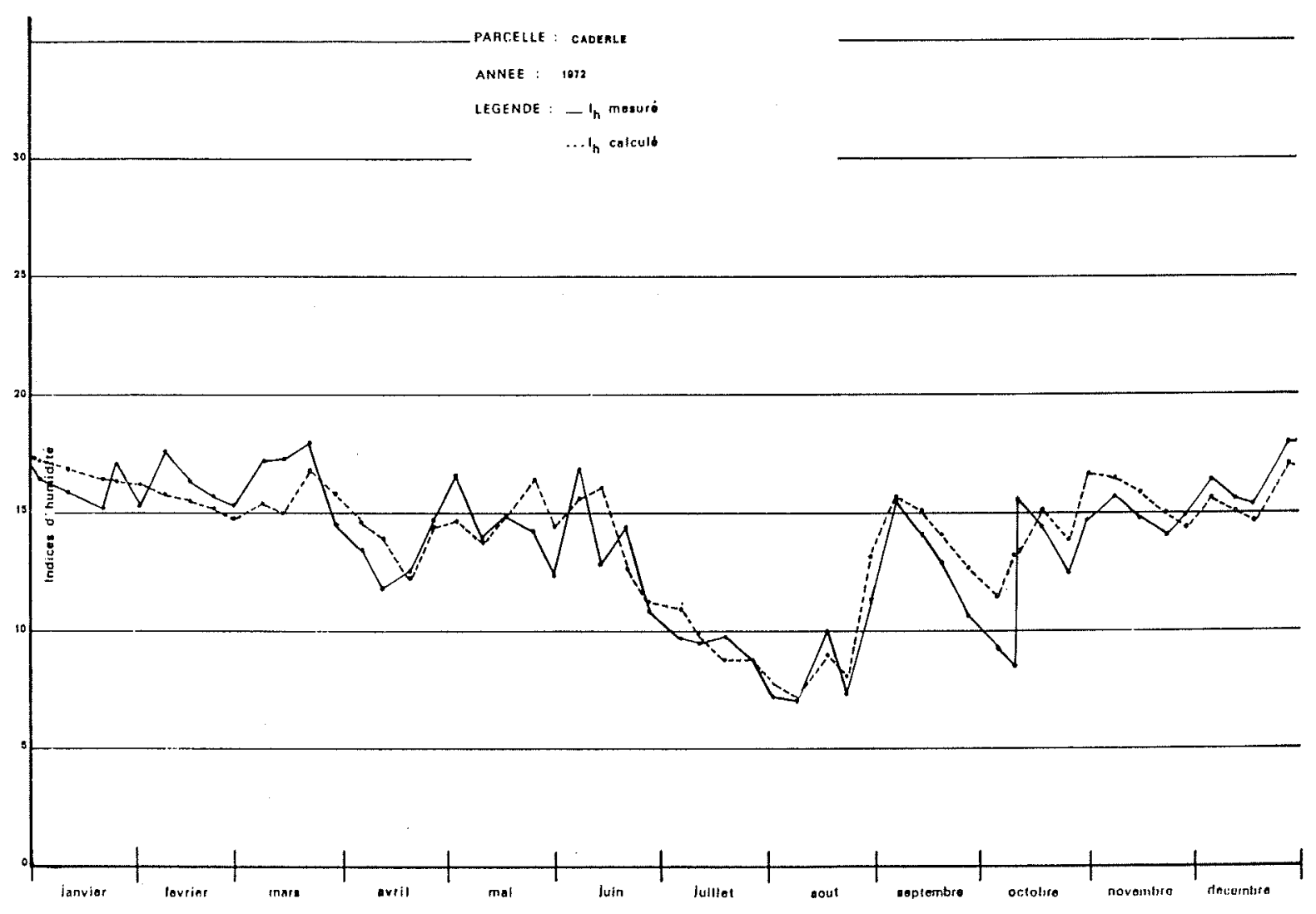

Figure 3

BCARTS RATRE LES INDICES CAICULES

EN LES IKDICES MESURES

Parcel1e CADRris

\begin{tabular}{|c|c|c|c|c|c|c|c|}
\hline \multirow{2}{*}{ AMRES } & \multirow{2}{*}{ TRIKESTRB } & \multicolumn{3}{|c|}{ Noyonno dos dcarta trimestriols } & \multicolumn{3}{|c|}{ Koyonno des ecarts ansue 1s } \\
\hline & & poultifs & ${ }^{6} \operatorname{ges}^{2}+15 \mathrm{~s}$ & 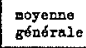 & pogitifa & négatifs & $\begin{array}{l}\text { moyenno } \\
\text { génersio }\end{array}$ \\
\hline 1970 & $\begin{array}{l}1 \\
2 \\
3 \\
4\end{array}$ & & & & & & \\
\hline 1971 & $\begin{array}{l}1 \\
2 \\
3 \\
4\end{array}$ & $\begin{array}{l}1,45 \\
2,11 \\
2,58 \\
2,02\end{array}$ & $\begin{array}{l}0,57 \\
1,39 \\
2,41 \\
0,52\end{array}$ & $\begin{array}{r}0,44 \\
1,03 \\
-0,87 \\
0,89\end{array}$ & 2,13 & 1,71 & 0,35 \\
\hline 1972 & $\begin{array}{l}1 \\
2 \\
3 \\
4\end{array}$ & $\begin{array}{l}1,19 \\
0,92 \\
0,80 \\
0,89\end{array}$ & $\begin{array}{l}1,09 \\
1,64 \\
0,89 \\
1,80\end{array}$ & 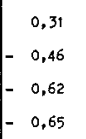 & $:, \infty$ & 1,33 & $-0,36$ \\
\hline 1973 & $\begin{array}{l}1 \\
2 \\
3 \\
4\end{array}$ & $\begin{array}{l}1,04 \\
2,40 \\
1,09 \\
0,43\end{array}$ & $\begin{array}{l}0,95 \\
2,12 \\
1,91 \\
1,01\end{array}$ & $\left\{\begin{array}{r}0,43 \\
-\quad 1,08 \\
-1,45 \\
-0,57\end{array}\right.$ & 1,14 & 1.62 & -0.67 \\
\hline 1974 & $\begin{array}{l}1 \\
2 \\
3 \\
4\end{array}$ & $\begin{array}{l}1,60 \\
1,58 \\
0,99 \\
1,77\end{array}$ & $\begin{array}{l}0,31 \\
0,91 \\
1,75 \\
1,23\end{array}$ & {$\left[\begin{array}{l}1,28 \\
-0,24 \\
-1,12 \\
-0,16\end{array}\right.$} & 1,56 & 1,27 & 0,03 \\
\hline
\end{tabular}

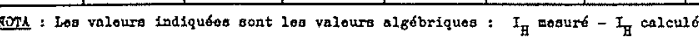

Figure 4
Les courbes donnant la comparaison des indices calculés aux indices mesurés montrent que la méthode définie ci-dessus traduit relativement bien le phénomène de variation dans le temps des indices.

Dans l'expérience effectuée, cette méthode conduit à des écarts moyens relativement faibles, puisque ces écarts varient à l'échelle de l'année

- pour la parcelle du POMPIDOU : de - 0,2 à - 2,5 points d'indice d'humidité.

- pour la parcelle de PALLIERES : de - 1,3 à + 3,5 points d'indice d'humidité.

- pour la parcelle de CADERLE : de $-0,7$ à +0,3 point d'indice d'humidité (voir tableau fíg. 4).

A l'échelle du trimestre, les écarts atteignent des valeurs un peu supérieures mais restent relativement faibles, les écarts maximaux observés étant pour les 3 parcelles ci-dessus de $-5,3,+4,7$ et $-1,4$. 


\section{Abstract \\ Calculation of soil moisture indexes in the Gardon d'Anduze catchment area from meteorological data}

The object of this study was to attempt to calculate soil moisture indexes from observed rainfall and evaporation data. The considered data had been collected by $M$. Hofer over a period of four years (1971-1974) for a flood-forecasting model of the Gardon d'Anduze catchment area in the Gard Department of France. During this period, the soil moisture index was measured once weekly at various points of the catchment area by taking core samples from the soil surface.

Rainfall and evaporation observations provided daily data which were used to calculate daily moisture indexes, each one of which was taken as basic data for calculating the next index from observed rainfall and evaporation over a period of 24 hours.

The following daily factors are taken into account in calculating the daily index-correction factor:

- An increase in moisture expressed as the percentage of rainfall actually stored in the top $20 \mathrm{~cm}$ soil layer, varying from 0 for satured soil to 0.9 for dry soil, based on a peak daily rainfall figure of $30 \mathrm{~mm}$.
- A decrease in moisture expressed as a percentage of measured evaporation, varying from 0 for dry soil to 0.7 and 0.95 for saturated soil in summer and winter respectively.

Comparison of the calculated and measured soil moisture indexes confirms the validity of the calculation for a general approach. Variations of mean deviation over a year are as follows :

-0.2 to -2.5 index points for one field with extreme indexes of 8.5 and 39 .

-1.3 to +3.5 index points for a second field with extreme indexes of 15 and 40 .

-0.7 to +0.3 index points for a third field with extreme indexes of 6 and 20 .

Mean deviations on a quarterly scale for the above fields do not exceed $-5.3,+4.7$ and -1.4 index points respectively. 